\title{
Quantitative Diclofenac-Analyse mittels gentechnisch veränderter Saccharomyces cerevisiae
}

\author{
Alfred Kick ${ }^{1}$, Maximilian Schulz ${ }^{1}$, Christine Schirmer ${ }^{1}$, Kai Ostermann ${ }^{2}$, Gerhard Rödel $^{2}$ und Michael Mertig $^{1,3}$ \\ ${ }^{1}$ Kurt-Schwabe-Institut für Mess- und Sensortechnik e.V. Meinsberg, Waldheim/Deutschland \\ ${ }^{2}$ Institut für Genetik, Technische Universität Dresden, Dresden/Deutschland \\ ${ }^{3}$ Institut für Physikalische Chemie, Technische Universität Dresden/Dresden, Deutschland \\ Kontakt: michael.mertig@ksi-meinsberg.de
}

\section{Einleitung}

Arzneimittelrückstände im Wasserkreislauf stellen ein weltweit wachsendes Problem dar. Die EUKommission hat daher eine Beobachtungsliste zur EU-Wasserrahmenrichtlinie herausgegeben, auf der auch der Wirkstoff Diclofenac (Abb. 1), der als Entzündungshemmer bzw. Antirheumatikum verwendet wird [1], gesetzt wurde. Es gibt Berichte über Diclofenac-Konzentrationen in Krankenhausabwässern von bis zu $7 \mu \mathrm{g} / \mathrm{l}(0,02 \mu \mathrm{mol} / \mathrm{l})$ und in Abwässern der pharmazeutischen Industrie von bis $200 \mu \mathrm{g} / \mathrm{l}(0,68$ $\mu \mathrm{mol} / \mathrm{l})[2]$. Aber bereits weitaus geringere Diclofenac-Konzentrationen können beispielsweise zu Schädigungen der Kiemen und Nieren bei Fischen führen [3]<smiles>O=C(O)Cc1ccccc1Nc1c(Cl)cccc1Cl</smiles>

Abb. 1: Strukturformel von Diclofenac.

Wir haben untersucht, wie genetisch veränderte Hefen (Saccharomyces cerevisiae) in einem lagerfähigen Assay in 96-Well-Mikrotiterplatten zur quantitativen Analyse von Diclofenac verwendet werden können. Die gentechnisch veränderten Hefezellen bilden, bei Anwesenheit von Diclofenac, verstärkt das Fluoreszenzprotein tRFP (turbo red fluorescent protein) [4-6]. Dadurch kann die biologische (toxische) Wirkung von Diclofenac direkt und unmittelbar erfasst werden. Die biologische Aktivität/Toxizität resultiert aus der Interaktion aller Inhaltsstoffe des Mediums einschließlich aller Metabolite, was durch eine rein physikalisch-chemische Analyse nicht erfasst werden kann.

\section{Methoden und Materialien}

Nährmedium und genetisch modifizierter Hefestamm Es wurde der Stamm Saccharomyces cerevisiae BY4741 p426-PDR5-tRFP verwendet. Dieser
Stamm wurde durch Transformation des Stammes Saccharomyces cerevisiae BY4741 (MATa; his3 $\Delta 1$; leu2 $\Delta 0$; met15 $\Delta 0$; ura3 $\Delta 0$ ) erhalten und produziert das rot fluoreszierende Protein tRFP bei Anwesenheit von Diclofenac [4].

Als Selektionsmarker wird Uracil verwendet. Transformanden können nur dann Uracil bilden, wenn sie das entsprechende Plasmid (p426-PDR5-tRFP) enthalten. In einem Nährmedium ohne Uracil können also nur diejenigen Zellen überleben, denen das Plasmid die Synthese des Uracils und damit auch des tRFP ermöglicht. Die Bildung von tRFP wird durch Diclofenac induziert bzw. verstärkt.

Das Nährmedium enthält 6,9 g/l Yeast nitrogen base (Formedium, UK) mit $5 \mathrm{~g} / \mathrm{l}$ Ammoniumsulfat, 1,926 $\mathrm{g} / \mathrm{l}$ Kaisers Mixture complete ohne Uracil (Formedium, UK) und $20 \mathrm{~g} / \mathrm{l}$ Glucose. Zur Herstellung des Mediums wurden $500 \mathrm{ml}$ zweifach konzentrierte Stammlösungen von Yeast nitrogen base und Kaisers Mixture complete ohne Uracil separat von einer $500 \mathrm{ml}$ Glucose-Stammlösung (40 g/l) autoklaviert und anschließend gemischt.

\section{Vorkulturen}

Für die Langzeitlagerung wurden je $500 \mu$ Z Zellsuspension ( $18 \mathrm{~h}$ alte Kultur, bei $30^{\circ} \mathrm{C}$ im Schüttler bei $150 \mathrm{rpm}$ ) mit $500 \mu \mathrm{l}$ einer $30 \%$ igen (v/v) Glycerinlösung gemischt und bei $-80{ }^{\circ} \mathrm{C}$ eingefroren (Kryocontainer, Nalgene ${ }^{\circledR}$ Mr. Frosty ${ }^{\circledR}$, Thermo Fisher Scientific MrFrosty ${ }^{T M}$ ). Damit wurden Vorkulturen des Hefestammes hergestellt, indem $1 \mathrm{ml}$ der Kryokultur als Inokulum zu $15 \mathrm{ml}$ Nährmedium gegeben wurde. Diese Vorkultur wurde $18 \mathrm{~h}$ bei $30^{\circ} \mathrm{C}$ im Schüttler inkubiert. Danach wurde die Vorkultur zweimal durch Zentrifugieren (3000 rcf, $7 \mathrm{~min}$ ), Überstand abnehmen und suspendieren in $15 \mathrm{ml}$ des frischen Nährmediums gewaschen. Die Trübung wurde bei $600 \mathrm{~nm}\left(\mathrm{OD}_{600}\right)$ mit einem Spektrophotometer (Nanodrop 2000c, Thermo Fisher Scientific) kontrolliert bzw. eingestellt.

Vorbereitung der lagerfähigen Assays in Mikrotiterplatten

Um lagerfähige Assays herzustellen, welche möglichst schnell zur Bestimmung der DiclofenacKonzentrationen in Probelösungen verwendet werden können, wurden 96-Well-Mikrotiterplatten (BRAND plates $^{\circledR}$ - cell Grade ${ }^{\text {TM }}$ premium BRAND 
$\mathrm{GmbH} \&$ Co KG, U-Boden) mit je $100 \mu \mathrm{l}$ Zellsuspension einer mit frischem Nährmedium gewaschenen Vorkultur befüllt. Die Trübung wurde auf $\mathrm{OD}_{600}=4$ eingestellt. Diese Platten wurden bei $-80^{\circ} \mathrm{C}$ über 24 $\mathrm{h}$ eingefroren (Kühlrate: etwa $1{ }^{\circ} \mathrm{C} / \mathrm{min}$ in Styroporbehälter), danach vakuumverpackt und 14 Tage bei $-20{ }^{\circ} \mathrm{C}$ gelagert.

Vor der Verwendung wurden die Platten auf Raumtemperatur gebracht (etwa $1 \mathrm{~h}$ ). Dabei kommt es zur Entgasung der Lösungen in den Wells. Um die Bläschen zu entfernen, wurden die Platten anschließend $4 \mathrm{~min}$ bei $1500 \mathrm{~g}$ zentrifugiert.

Danach wurden je $100 \mu \mathrm{l}$ der Proben bzw. Kalibrierstandards $(0,0,25,0,50,0,751,00,1,25,1,50,1,75$ und $2 \mu \mathrm{M}$ Diclofenac in Nährmedium) dazugegeben. Zur Herstellung einer Diclofenac-Stammlösung wurde Diclofenac-Natriumsalz (Sigma Aldrich) in $50 \% \mathrm{i}-$ gem Ethanol (v/v) gelöst, um eine $20 \mathrm{mM}$ Lösung zu erhalten. Anschließend wurde eine $50 \mu \mathrm{M}$ Stammlösung durch Verdünnen im Nährmedium hergestellt. Aus dieser Stammlösung wurden weitere Verdünnungen hergestellt, von denen je $100 \mu \mathrm{l} z u$ den Zellsuspensionen in der Mikrotiterplatte gegeben wurden. Danach wurden die Platten mit einer Abdeckmembran (Breathe-EASIER ${ }^{\mathrm{TM}}$, Diversified Biotech) verschlossen, um eine möglichst gute Sauerstoffversorgung der Zellen zu gewährleisten.

\section{Fluoreszenzmessungen}

Die Fluoreszenzmessungen der vorbereiteten Mikrotiterplatten erfolgte in einem Mikroplatten-Reader (Synergy ${ }^{\mathrm{TM}} \mathrm{H} 1$ BioTek Instruments, Inc., Winooski, USA). Die Temperatur wurde auf $30^{\circ} \mathrm{C}$ eingestellt und kontrolliert. Zwischen den Fluoreszenzmessungen wurde die Platte im Reader geschüttelt (Doppelorbital, $528 \mathrm{rpm}$ ). Die Fluoreszenzanregung fand bei $532 \pm 9 \mathrm{~nm}$ statt und die Emission wurde bei $600 \pm 9 \mathrm{~nm}$ aufgezeichnet. Fluoreszenzanregung und Auslesung der Emission erfolgten durch den Boden der Mikrotiterplatte.

\section{Ergebnisse und Diskussion}

In Abbildung 2 ist der zeitliche Verlauf der Fluoreszenzintensität in drei ausgewählten Wells der niedrigsten Diclofenac-Konzentrationen gezeigt. Man erkennt, dass nach etwa $6 \mathrm{~h}$ die Bildung des tRFP einsetzt und nach etwa $16 \mathrm{~h}$ das Maximum erreicht. Dies geschieht auch in der Kontrolle ohne Diclofenac, da der Promotor, unter dessen Kontrolle das tRFP-Gen steht, immer eine basale Expression zulässt. Die Synthese des tRFP findet auch in Diclofenac-freiem Medium statt und muss bei der Quantifizierung beachtet werden, indem möglichst ein Standardadditionsverfahren angewendet wird. Dabei sollten die Kalibrierlösungen in die Probenlösung gegeben werden, um Matrixeffekte zu berücksichtigen. Für die Kalibrierung ist auch eine Probe not- wendig, welche die komplette Probenmatrix, aber kein Diclofenac enthält.

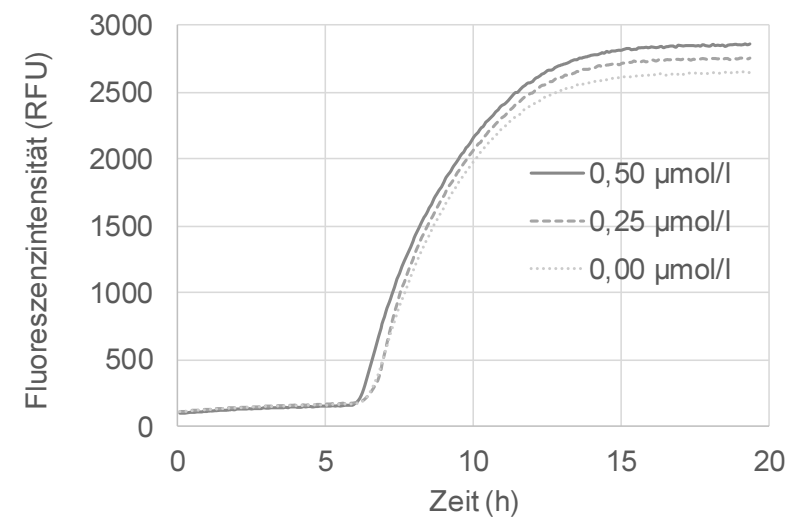

Abb. 2: Kinetik der Fluoreszenzintensitäten in Abhängigkeit von der Diclofenac-Konzentration. Drei ausgewählte Wells im niedrigen Konzentrationsbereich.

Außerdem konnte festgestellt werden, dass eine Mehrfachbestimmung mit mindestens 3 bis 4 Wells pro Diclofenac-Konzentration notwendig ist, um eine signifikante Erhöhung der Fluoreszenzintensität $a b$ $0,25 \mu \mathrm{M}$ festzustellen und eine Kalibrierung vorzunehmen (Abb. 3).

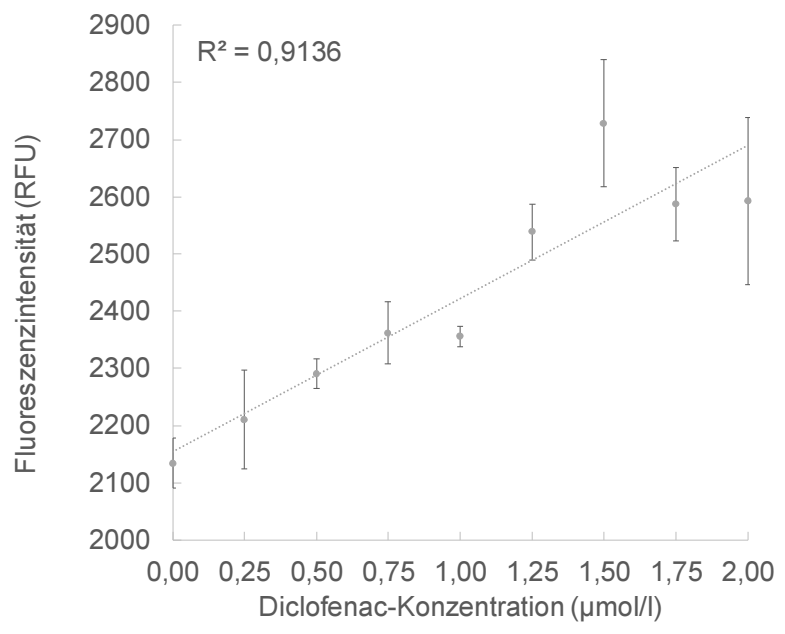

Abb. 3: Fluoreszenzintensitäten nach $12 \mathrm{~h}$ in $\mathrm{Ab}$ hängigkeit von der Diclofenac-Konzentration. Mittelwerte und Standardabweichungen aus je 3 oder 4 Wells.

\section{Zusammenfassung und Ausblick}

Es konnte ein lagerfähiger Assay zur quantitativen Bestimmung von Diclofenac mittels gentechnisch veränderter Saccharomyces cerevisiae entwickelt werden. In Mikrotiterplatten und einem FluoreszenzPlattenreader wurde eine Nachweisgrenze von $0,2 \mu \mathrm{mol} / /$ Diclofenac im definierten Nährmedium etwa $12 \mathrm{~h}$ nach der Zugabe des Analyten erzielt.

Um die Zuverlässigkeit des Assays zu erhöhen, sollen entsprechende Gene des Plasmids genomisch integriert werden. Dadurch soll vermieden werden, 
dass Variationen der Plasmid-Anzahl pro Zelle auftreten und so die Fluoreszenzintensität, trotz gleicher Diclofenac-Konzentration, ebenfalls stark variiert. Eine deutliche Vereinfachung der Quantifizierung ist zu erwarten, wenn es gelingt einen anderen Promoter zu identifizieren, der keine basale Expression zulässt und somit eine tRFP-Synthese nur in Anwesenheit von Diclofenac stattfinden kann. Damit wäre es nicht notwendig, eine Probenmatrix bereitzustellen die kein Diclofenac enthalten darf aber alle anderen Bestandteile der zu untersuchenden Lösung enthält. Versuche an realen Abwässern müssen zeigen, wie stark Matrixeffekte die Sensitivität des Assays beeinflussen.

\section{Literatur}

[1] Richtlinie 2013/39/EU des Europäischen Parlaments und des Rates zur Änderung der Richtlinien 2000/60/EG und 2008/105/EG in Bezug auf prioritäre Stoffe im Bereich der Wasserpolitik, (2013)

[2] VIENO, N.; SILLANPÄÄ, M.: Fate of diclofenac in municipal wastewater treatment plant - A review. In: Environment International 69 (2014) S. 28-39

[3] L.A. PÉREZ-ESTRADA, S. MALATO, W. GERNJAK, A. AGÜERA, E.M. THURMAN, I. FERRER, A.R. FERNÁNDEZ-ALBA, Environ. Sci. Technol. 39 (2005) S. 8300-8306

[4] SCHULLER, A.; RÖDEL, G.; OSTERMANN, K.: Tuning the sensitivity of the PDR5 promoter-based detection of diclofenac in yeast biosensors. In: Sensors 17 (2017), S. 1506-1522

[5] SCHIRMER, C.; POSSECKARDT, J.; KICK, A.; REBATSCHEK, K.; FICHTNER, W.; OSTERMANN, K.; SCHULLER, A.; RÖDEL, G.; MERTIG, M.: Encapsulating genetically modified Saccharomyces cerevisiae cells in a flow-through device towards the detection of diclofenac in wastewater. In: J Biotechnol 284 (2018), S. $75-83$

[6] SCHIRMER, C.; POSSECKARDT, J.; SCHRÖDER, M.; GLÄSER, M.; HOWITZ, S.; SCHARFF, W.; MERTIG, M.: Portable and low-cost biosensor towards onsite detection of diclofenac in wastewater. In: Talanta 203 (2019), 242-247

\section{Danksagung}

Das Kurt-Schwabe-Institut für Mess- und Sensortechnik Meinsberg e.V. wird mitfinanziert durch Steuermittel auf der Grundlage des vom Sächsischen Landtag beschlossenen Haushaltes. 